\title{
Anticytokine approaches in pulmonary fibrosis: bringing factors into focus
}

\author{
R K Coker, G J Laurent
}

\begin{abstract}
The prognosis of pulmonary fibrosis is poor and current therapies inadequate. Recent progress in understanding the mechanisms underlying the pathogenesis of this disease leads us to expect that inhibitors of cytokine and polypeptide growth factor will provide novel therapeutic agents. This paper outlines the role of cytokines in the pathogenesis of pulmonary fibrosis and concludes that there are compelling reasons to explore anticytokine therapeutic approaches. It also proposes criteria that will enable us to evaluate such agents in vivo and suggests approaches which might overcome the obstacles presented by control mechanisms which recruit a multiplicity of factors.
\end{abstract}

(Thorax 1997;52:294-296)

Keywords: pulmonary fibrosis, anticytokine.

Pulmonary fibrosis can follow diverse insults including exposure to inhaled dusts, radiation, and certain drugs. It may complicate local or systemic pathology including pulmonary infections, sarcoidosis, and connective tissue disorders. Diffuse pulmonary fibrosis arising with no known predisposing factors is termed cryptogenic fibrosing alveolitis (idiopathic pulmonary fibrosis in the USA).

The UK prevalence of cryptogenic fibrosing alveolitis (CFA) alone is approximately five people in every 100000 . Annual mortality, around 1400, has more than doubled since 1980. Current treatment comprises corticosteroids and immunosuppressants but most clinical series show only $50 \%$ five year survival despite treatment. Recognition of the poor prognosis and long term ineffectiveness of treatment with potentially serious side effects has led many respiratory physicians to adopt conservative approaches to management.

The last decade has generated significant advances in our understanding of the pathogenesis of the condition. One current hypothesis is that polypeptide mediators or cytokines, released by resident lung cells and recruited inflammatory cells, stimulate fibroblast replication and increased synthesis of extracellular matrix proteins including collagen. Collagen deposition then impedes gas exchange. As key cytokines involved in this process are elucidated, anticytokine therapy is emerging as a promising prospect.

\section{Scientific basis}

Alveolar epithelial and/or capillary cell injury occurs early in pathogenesis (fig 1). Circulating immune cells, including monocytes, neutrophils, lymphocytes and eosinophils, are then recruited into the lung. These effector cells release cytokines which stimulate target cells typically fibroblasts - to replicate and synthesise increased amounts of collagen. Resident lung

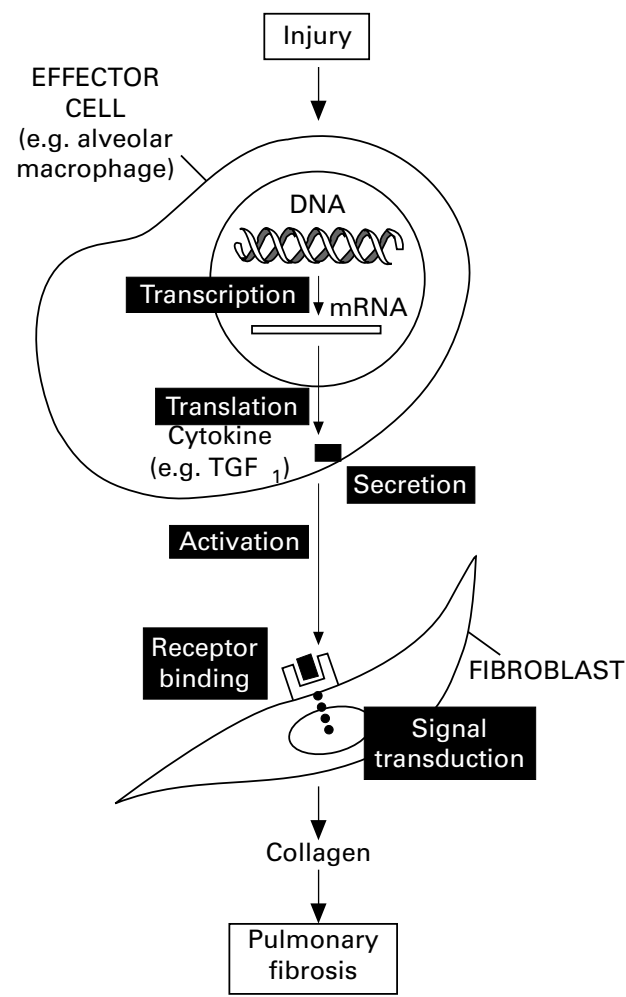

Figure 1 Potential targets for anticytokine therapy in pulmonary fibrosis. Therapeutic agents could be designed to inhibit any of the stages highlighted in black. Injury promotes cytokine gene transcription in the nuclei of effector cells such as alveolar macrophages. The $m R N A$ transcript then undergoes translation in the cytoplasm to generate a polypeptide product. After its secretion outside the cell the cytokine may undergo activation prior to receptor binding on target cells such as fibroblasts. Signal transduction results in enhanced collagen synthesis and consequent fibrosis. 
cells, including macrophages, alveolar epithelial and endothelial cells, can also release such mediators.

Various cytokines have been implicated in the pathogenesis of pulmonary fibrosis, including transforming growth factor $\beta_{1}$ (TGF- $\left.\beta_{1}\right)$, tumour necrosis factor $\alpha$ (TNF- $\alpha$ ), plateletderived growth factor (PDGF), insulin-like growth factor 1 (IGF-1), and the interleukins IL-1 and IL-8. ${ }^{1}$ Recently interest has also focused on endothelin 1 (ET-1) ${ }^{2}$ and bloodderived proteins including thrombin. ${ }^{3}$

Of those investigated so far, the TGF- $\beta$ family has the most potent stimulatory effect on extracellular matrix deposition. TGF- $\beta_{1}$, secreted as a latent precursor, stimulates fibroblast procollagen gene expression and protein synthesis. It also inhibits collagen breakdown. Our data suggest that the other mammalian TGF- $\beta$ isoforms, TGF- $\beta_{2}$ and TGF- $\beta_{3}$, also stimulate human lung fibroblast collagen synthesis and reduce degradation. ${ }^{4}$ In experimental lung fibrosis enhanced TGF- $\beta_{1}$ gene expression precedes increased collagen expression. TGF$\beta_{1}$ antibodies attenuate murine bleomycin-induced lung fibrosis, and human fibrotic lung tissue shows increased TGF- $\beta_{1}$ gene and protein expression.

There is also evidence to implicate tumour necrosis factor $\alpha(\mathrm{TNF}-\alpha)$ in the pathogenesis of pulmonary fibrosis. It can promote fibroblast replication and collagen synthesis, and lung $\mathrm{TNF}-\alpha$ gene expression increases in mice following bleomycin administration. Soluble TNF- $\alpha$ receptors attenuate fibrosis in murine models of lung fibrosis, ${ }^{5}$ bronchoalveolar lavage fluid derived macrophages from patients with CFA or asbestosis release increased amounts of TNF- $\alpha$, and transgenic mice overexpressing $\mathrm{TNF}-\alpha$ in alveolar epithelium develop pulmonary fibrosis. ${ }^{6}$

Diminished production of potentially antifibrotic cytokines such as interferon (IFN) gamma may also enhance collagen deposition in pulmonary fibrosis ${ }^{7}$ and various physical, chemical and hormonal agents, probably interacting with cytokines, are now recognised to modulate collagen production. Consequently cytokines can interact in vivo to produce different effects from those observed in vitro and target cell phenotype may modify the response. It may therefore be useful to establish criteria for key fibrogenic cytokines, enabling us to select and develop appropriate anticytokine treatments for patients with pulmonary fibrosis. Inspired by Koch's postulates we propose that the following criteria should be satisfied before anticytokine therapy is indicated: (1) a candidate cytokine (or polypeptide growth factor) should stimulate fibroblast replication or procollagen production; (2) mediator gene expression and protein production should increase in the lungs of patients with pulmonary fibrosis; and (3) inhibitors of mediator function should attenuate fibrosis in animal models of the disease.

TNF- $\alpha$ and TGF- $\beta_{1}$ are currently the only cytokines that fulfil all three criteria. This information provides a rational basis for developing anticytokine agents and strongly suggests that anti-TNF- $\alpha$ and anti-TGF- $\beta_{1}$ therapy should now be evaluated in patients with pulmonary fibrosis. In the future additional cytokines may emerge that satisfy these criteria.

\section{Mechanisms of anticytokine therapy}

Anticytokine therapeutic strategies should logically target one or more key events in cytokine synthesis and receptor binding (fig 1). DNA transcription generates an mRNA transcript whose translation results in polypeptide assembly. Agents can inhibit protein production in several ways. Antisense oligodeoxynucleotides may hybridise with nuclear mRNA, activating RNases. They can also bind cytoplasmic mRNA, inhibiting ribosomal activity through steric hindrance. Alternatively, ribozymes (RNA molecules with enzymatic activity) can prevent translation by binding and cleaving the mRNA transcript in the cytoplasm.

TGF- $\beta_{1}$ activation requires binding to the mannose-6-phosphate receptor; agents competing for binding may therefore be antifibrotic. Inhibitors of growth factor-receptor binding on target cells include antibodies, soluble receptors, receptor antagonists, and molecules which remove the cytokine from its site of action. Elucidation of signal transduction pathways within the fibroblast may promote development of compounds which uncouple individual cytokine signalling pathways and block specific effects of profibrotic mediators.

\section{Therapeutic potential}

ANTICYTOKINE STRATEGIES UNDER EVALUATION The following illustrations demonstrate the potential of anticytokine strategies as novel treatments for pulmonary fibrosis. TGF- $\beta_{1}$ antisense reduces arterial wall proteoglycan synthesis ${ }^{8}$ and can eradicate established intracranial rat gliomas. ${ }^{9}$ Mannose-6-phosphate competes with TGF- $\beta$ for binding to the mannose-6-phosphate receptor, preventing TGF- $\beta$ activation, and is under evaluation in clinical trials to reduce cutaneous wound scarring.

Anti-TNF antibodies reduce inflammatory markers in rheumatoid arthritis ${ }^{10}$ but immunogenicity may limit use of cytokine antibodies in pulmonary fibrosis where prolonged treatment will probably be required. Soluble cytokine receptors or binding proteins may therefore provide a safer alternative. Administration of the proteoglycan decorin, a natural inhibitor of TGF- $\beta$, attenuates fibrosis in an animal model of glomerulonephritis ${ }^{11}$ as does gene therapy upregulating decorin expression. ${ }^{12}$ Receptor antagonists effective in vitro are now available for TGF- $\beta_{1}, \mathrm{ET}-1$, and IL-1 but their value in fibrosis remains to be investigated.

OVERCOMING MULTIPLE GROWTH FACTORS Multiple cytokines and polypeptide growth factors are likely to be implicated in the pathogenesis of pulmonary fibrosis. Their functions may overlap and eliminating one may be in- 
effective therapeutically as another takes over its role. Possible solutions include blocking the actions of several cytokines simultaneously or inhibiting the actions of individual mediators at different stages of the disease process. Another approach may be to identify and block intracellular signalling pathways common to several cytokines.

CLINICAL TRIALS

There is an urgent need for clinical trials of new therapies for this disease. Although different initiating events and cytokines may be implicated in different diseases, such as CFA and sarcoidosis, there is currently no evidence that the process of fibrogenesis differs significantly between diseases. Anticytokine strategies should therefore be applicable in various settings and could be used concurrently with other treatments, new or established. Short to medium term outcome measures will be required for pharmaceutical collaboration. These could realistically include bronchoalveolar lavage procollagen peptide levels, a marker of collagen synthesis, and lung function including assessment of the alveolar/arterial gradient at rest and on exertion. Other markers of fibrosis may become available in the future as research continues.

\section{Conclusion}

There is strong evidence implicating several cytokines including TGF- $\beta_{1}$ and TNF- $\alpha$ in the pathogenesis of pulmonary fibrosis. Additional mediators are becoming implicated as research progresses, including blood-borne mediators and products of the coagulation cascade. Recognition of key profibrotic cytokines has facilitated consideration of anticytokine thera- peutic strategies, and establishing criteria for profibrotic growth factors may guide selection of appropriate anticytokine therapy. The diversity of approaches and successful application in other fields suggest there is potential for developing novel anticytokine drugs to treat pulmonary fibrosis. Continued research is critical, especially into ways of targeting multiple growth factors, but now is the time to begin evaluating these agents in clinical trials.

1 Coker RK, Laurent GJ. Pathogenesis of pulmonary fibrosis: implications for pharmacological intervention. In: Walte $\mathrm{EH}, \mathrm{du}$ Bois RM, eds. Immunology and management of interstitial lung disease. London: Chapman and Hall, 1995 19-36.

2 Cambrey A, Harrison NK, Dawes KE, Southcott AM, Black CM, du Bois RM, et al. Increased levels of endothelin-1 in bronchoalveolar lavage fluid from patients with systemic sclerosis contribute to fibroblast mitogenic activity in vitro. Am $\mathcal{F}$ Respir Cell Mol Biol 1994;11:439-45.

3 Hernandez-Rodriguez NA, Cambrey AD, Harrison NK, Chambers RC, Gray AJ, Southcott AM, et al. Role of thrombin in pulmonary fibrosis. Lancet 1995;346:1071-3.

4 Coker RK, Laurent GJ, Shahzeidi S, Lympany PA, du Bois RM, Jeffery PK, et al. Transforming growth factor $\beta_{1},-\beta_{2}$ RM, Jeffery PK, et al. Transforming growth factor $\beta_{1},-\beta_{2}$ vitro but are differentially expressed during bleomycinvitro but are differentially expressed during bleom

5 Piguet PF, Vesin C. Treatment by human recombinant soluble TNF receptor of pulmonary fibrosis induced by soluble TNF receptor of pulmonary fibrosis induced by

6 Miyazaki Y, Araki K, Vesin C, Garcia I, Kapanci Y, Whitset $\mathrm{JA}$, et al. Expression of a tumor necrosis factor- $\alpha$ transgene in murine lung causes lymphocytic and fibrosing alveolitis. f Clin Invest 1995;96:250-9.

7 Prior C, Haslam P. In vivo levels and in vitro production of interferon gamma in fibrosing interstitial lung diseases. Clin Exp Immunol 1992;88:280-7.

8 Merrilees MJ, Scott L. Antisense s-oligonucleotide against transforming growth factor- $\beta_{1}$ inhibits proteoglycan synthesis in arterial wall. $\mathcal{F}$ Vasc Res 1994;31:322-9.

9 Fakhrai H, Dorigo D, Shawler DL, Lin H, Mercola D, Black KL, et al. Eradication of established intracranial rat gliomas by transforming growth factor $\beta$ antisense gen therapy. Proc Natl Acad Sci USA 1996;93:2909-14.

10 Elliott MJ, Maini RN, Feldmann M, Long-Fox A, Charles P, Katsikis P, et al. Treatment of rheumatoid arthritis with chimeric monoclonal antibodies to tumor necrosis factor a. Arthritis Rheum 1993;36:1681-90.

11 Border WA, Noble NA, Yamamoto T, Harper JR, Yamaguch $\mathrm{Y}$, Pierschbacher MD, et al. Natural inhibitor of transforming growth factor- $\beta$ protects against scarring in perimental kidney disease. Nature 1992;360:361-4

12 Isaka Y, Breese DK, Ikegaya K, Kaneda Y, Imai E, Noble $\mathrm{NA}$, et al. Gene therapy by skeletal muscle expression of decorin prevents fibrotic disease in rat kidney. Nature Med decorin prevent 